\title{
ZWPS: A Hybrid Selection Technique for Small Target Acquisition in Pen-Based Interfaces
}

\author{
Jibin Yin and Xiangshi Ren \\ Kochi University of Technology, Kochi 782-8502, Japan \\ 088402 e@gs.kochi-tech.ac.jp, ren.xiangshi@kochi-tech.ac.jp
}

\begin{abstract}
In this paper a novel zoom-based technique with pressure (hereafter referred to as "ZWPS") is proposed to improve small target selection in penbased interfaces. In this technique pressure is used as a switch mode to couple a standard pointing technique and a zoomable technique together. ZWPS allows both precise and normal selections. We conducted an experiment to examine the effectiveness of ZWPS. The experimental results indicate that ZWPS significantly enhance small target selections.
\end{abstract}

Keywords: pen-based interface, pressure, zoom-based, small target acquisition.

\section{Introduction}

Pen-based devices allow direct touch and data manipulation on screen with stylus pens, which has a very strong appeal to users. However, being direct between control and display, screens of pen-based devices have special limitations. First, users generally suffer from a small amount of parallax error due to the display being slightly below the touch sensitive surface; second, users' finger, hand and arm can obscure part of the screen. The limitations, to some degree, bring difficulties to target selection tasks, especially for small target selections. There are some researches that have proposed techniques to improve small target selection performance $[1,3,4,5,6]$ in a pen-based environment. This issue was notably addressed by Sears, Shneiderman and colleagues $[3,5,6]$. Their basic technique, called Take-Off, provides a cursor above a pen-tip or the user's finger tip with a fixed offset when touching the screen to achieve precise selection.

Zoom pointing is also a typical technique for small target selection, which is currently used in many painting systems. For example, drawing a precise line from one dot (e.g., railway station) to another (e.g., a college) on a digital map requires the user to zoom in and click on one end, zoom out to find the other end, zoom in again to click on the other end, then zoom out again to look at both ends, all of which cause great inconvenience to users. Moreover, after zooming in, the user may lose information in the overview and the detailed windows. The switch between zooming in and zooming out often interrupts the user's attention. This awkward situation becomes our direct motivation to study alternatives to the current technique. We present ZWPS which integrates two techniques by using pressure as switch mode to allow both precise and normal selections. 


\section{ZWPS Design and Implementation}

We seek to find a selection technique that should satisfy the following conditions:

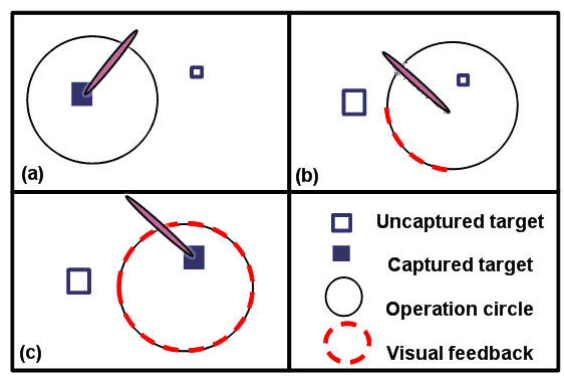

Fig. 1. A process for selecting a target using ZWPS: (a) shows the process of selecting a target of normal size in standard pointing mode. (b) and (c) illustrate the process of selecting a small target by zoom selection mode.

(1) have a zoom function to allow a small target selection; (2) Provide a flexible and seamless switch mode between zooming in and out; (3) zoom only a certain part of the display area to prevent the user from losing the information in overview.

Therefore, we present a novel technique called ZWPS to enhance small target selection. ZWPS, in essence, is a hybrid selection technique that includes two selection techniques: the standard pointing technique and the zoom technique. The standard pointing selection mode works in selecting common size targets while zoom selection mode works best when selecting small size targets.

To couple the two techniques in ZWPS a suitable switch mode is essential. The properties of pressure (easily producing discrete controlled states) make it competent to seamlessly switch modes in ZWPS. To determine a proper switch threshold, a pilot experiment with 10 subjects was performed. The task was to draw freehand strokes (arbitrary curves and straight lines), basic geometrical graphs (such as rectangles and circles) and a mixed set of Roman, Japanese, and Chinese (kanji) characters and signatures on a blank space in a natural manner. Pen-tip pressure was recorded in a $17 \mathrm{~ms}$ sampling periods. Ninety-five percent of the force samples fell within the 210 to 810 units range. The results showed that pressure levels of more than 810 units were seldom used in a natural manner. Therefore, in ZWPS the threshold value is set at 970 units.

An operation circle (see Figure 1) is also defined to specify a zoomed area. Targets that are completely enclosed by the circle can be zoomed in by imposing heavy pressure, enough to surpass the switch threshold. By conducting a pilot experiment, the diameter of the operational circle was set at $24 \mathrm{~mm}$. When more than one target is enclosed by the operation circle, they are zoomed in or out together simultaneously. The parts that are beyond its border can be squeezed into its edge.

An arc with a dotted red line, which is attached on the border of the operational circle, is employed as visual feedback to indicate the current pressure state. When the arc, augmented by pressure from a pen, increases to a complete circle (surpassing the 
threshold) it means that the zoom technique is activated, Conversely, ZWPS still maintains the standard pointing technique, when effective pressure is not applied.

As illustrated in Figure 1 when selecting a target of normal or big size, the user, imposing light pressure on a pen-tip, taps the target directly to select it in the standard selection mode (Figure 1a). When selecting a very small target the target is first enclosed (Figure 1b) by the operational circle, and then the zoom selection mode is activated by heavy pressure to enlarge the target. The pen-tip slides into the zoomed target to highlight it (Figure 1c). When the pen is lifted from the screen, the target is selected and it recovers to its original size. Note that ZWPS only zooms in the operation circle area around the cursor, not the whole area in the view window, which enables the user to avoid losing the information in overview, even when it is in zoomed status.

\section{Experiment}

We compare the performance of ZWPS to other techniques for selecting small targets. We chose Take-off, a promising technique, as the baseline, because it is a very common baseline in many other evaluations of techniques for small-target selection reported to date $[3,5,6]$.

Twelve subjects (11 male and 1 female) all with right-handed and previous experience using computers were tested for the experiment. The average age was 24.9 years.

\subsection{Procedure}

The experimental task was simple reciprocal target pointing. Two square targets of width $W$, separated by distance $D$ were presented on the screen. The goal target was colored red and underlined, which alternated between the two. Participants were instructed to select the red target as quickly and, more importantly, as accurately as possible. When a desired target was selected correctly a beep sound played.

The design of the experiment was as follows: crossed Technique $(T) \mathrm{x}$ Width $(W) \mathrm{x}$ Distance $(D)$. Since the focus of this study is precision pointing, target width W was set at 1,4 and 8 pixels and distance $D$ was set at $100,250,750$. For each of the two techniques, 9 combinations ( $=3$ target widths $\mathrm{x} 3$ distances) appeared in random order. Each subject had a total of 63 attempts (7 blocks x 3 widths $x 3$ distances).

\subsection{Results}

A repeated measures analysis of variance showed that there was a significant difference between Take-off and ZWPS in selection time, $F(1,47)=39.9, \mathrm{p}<.001$. Target size had a significant impact on mean selection time. There were also interaction effects between Techniques $x$ Target size for selection time, $F(2,47)=12.5$, $\mathrm{p}<.001$. ZWPS took less time than Take-off for 1, 4 and 8 pixels. The difference between them became more significant as targets became smaller. The results indicted that ZWPS delivered more benefits for small-target selections. 
In particular, for 1 pixel or 2 pixel targets, Take-off was dramatically more error prone than ZWPS. For the large target (8 pixels), Take-off was still higher than ZWPS in error rate.

Subjects gave ZWPS a significantly higher rating than Take-off. ZWPS enables the user to easily select targets by zooming them, especially for very small (pixel level) targets.

\section{Discussion}

ZWPS utilizes pressure as a switch mode to couple two selection techniques (a standard pointing technique and a zoom technique) to enable it to be competent for both normal and precise selections. An experiment was conducted comparing it with Take-off, a current promising technique. Experimental results indicate that ZWPS can bring significant advantages to small target selection performances. In fitts' law [2] study ZWPS roughly fits Fitts' law with low $\mathrm{R}^{2}=0.8$. Its reason is that when using ZWPS to select small targets the selection process is more complex than a typical single pointing task, which requires users to perform two steps, i.e., enlarge the target and select the goal target. In ZWPS pressure plays a successful state switch role, which suggests that pressure should be a good alternative to mode switch.

\section{References}

1. Albinsson, P.-A., Zhai, S.: High Precision Touch Screen Interaction. In: Proc. CHI 2003, pp. 105-112 (2003)

2. Fitts, P.M.: The information capacity of human motor system in controlling the amplitude of movement. Journal of Experimental Psychology 47, 381-391 (1954)

3. Potter, R.L., Weldon, L.J., Shneiderman, B.: Improving the accuracy of touch screens: an experimental evaluation of three strategies. In: Proc. CHI' 88, pp. 27-32 (1988)

4. Ren, X., Moriya, S.: Improving selection performance on pen-based system: A study of penbased interaction for selection tasks. ACM Trans. Computer-Human Interaction 7(3), 384-416 (2000)

5. Sears, A., Shneiderman, B.: High Precision Touchscreens: Design Strategies and Comparison with a Mouse. International Journal of Man-Machine Studies 43(4), 593-613 (1991)

6. Shneiderman, B.: Touch screens now offer compelling uses. IEEE Software 8(2), 93-94 (1991) 